\title{
Parameter regimes for slow, intermediate and fast MHD shocks
}

\author{
P. DELMONT $T^{1,2} \dagger$ and R. KEPPEN S $\mathrm{K}^{1,2,3,4}$ \\ ${ }^{1}$ Centre for Plasma Astrophysics, K.U.Leuven, Belgium \\ ${ }^{2}$ Leuven Mathematical Modeling and Computational Science Centre, K.U.Leuven, \\ Belgium \\ ${ }^{3}$ Astronomical Institute, Utrecht University, The Netherlands \\ ${ }^{4}$ FOM institute for Plasma Physics Rijnhuizen, Nieuwegein, The Netherlands
}

(Received today)

\begin{abstract}
We investigate under which parameter regimes the MHD RankineHugoniot conditions, which describe discontinuous solutions to the MHD equations, allow for slow, intermediate and fast shocks. We derive limiting values for the upstream and downstream shock parameters for which shocks of a given shock type can occur. We revisit this classical topic in nonlinear MHD dynamics, augmenting the recent time reversal duality finding by Goedbloed (2008) in the usual shock frame parametrization.
\end{abstract}

\section{Introduction}

\subsection{Intermediate shocks in $M H D$}

The dynamic behaviour of plasmas is described by the magnetohydrodynamic (MHD) equations, where a central role is played by the Alfvén speed. Discontinuous solutions only satisfy the integral form of the MHD equations, i.e. the Rankine-Hugoniot conditions (RH). These $\mathrm{RH}$ conditions have been studied extensively in established as well as more recent literature (see e.g. Germain (1960), Anderson (1963), Jeffrey \& Taniuti (1964), Liberman \& Velikhovich (1986), Sturtevant (1987), Gombosi (1998), Goedbloed (2008)) and just express the basic nonlinear conservation laws across a discontinuity. Many authors have since paid attention to MHD shock stability as well (amongst others Akhiezer et al. (1959)). The interaction of small perturbations with MHD (switch-on and switch-off) shocks was studied both analytically by Todd (1965) and numerically by Chu \& Taussig (1967). Later on, the evolutionarity of intermediate shocks, which cross the Alfvén speed, has been adressed. A discontinuity is said to be evolutionary when small perturbations imposed on it lead to an evolution that remains close to the initial discontinuity. According to classical stability analysis, intermediate shocks are not evolutionary so one should not obtain such shocks in physically realizable situations. On the other hand, Wu (1987), De Sterck et al. (1998) and others found intermediate shocks in one and two dimensional numerical simulations respectively. De Sterck \& Poedts (2000) were the first to find intermediate shocks in three dimensional numerical simulations. The evolutionary condition became controversial and Myong \& Roe

$\dagger$ Present e-mail address: peter.delmont@wis.kuleuven.be 
(1997(a,b)), amongst others, argue that the evolutionary condition is not relevant in dissipative MHD. Wu $(1988,1990)$ also argued that intermediate shocks are admissible. Shock observations in the heliosphere can be found in favor of their existence as well: Chao et al. (1993) reported a $2 \rightarrow 4$ intermediate shock observed by Voyager 1 in 1980 and Feng \& Wang (2008) recognised a $2 \rightarrow 3$ intermediate shock, which was observed by Voyager 2 in 1979.

One can also encounter compound waves in ideal MHD. These compound waves can consist of a slow shock which travels with its maximal propagation speed and a rarefaction fan directly attached to it. Brio \& Wu (1988) detected those compound waves in numerical simulations which have become classical test problems for numerical codes. Another type of compound signal consists of a slow shock layer, immediately followed by a rotational discontinuity (Whang et al. (1998)). The importance of compound waves is that they can be an alternative to intermediate shocks to cross the Alfvén speed. Barmin et al. (1996) argue that if the full set of MHD equations is used to solve planar MHD, a small tangential perturbation on the magnetic field vector splits the rotational jump from the non-evolutionary compound wave. This transforms the latter one into a slow shock, such that the compound wave is non-evolutionary. They investigate the reconstruction process of the non-evolutionary compound wave into evolutionary shocks. Falle \& Komissarov $(1997,2001)$ also reject intermediate shocks on evolutionary grounds, and suggest a shock capturing scheme based on Glimm's method (Glimm (1965)) for numerically obtaining purely evolutionary solutions in MHD. Our goal in this paper is to determine in which regions of parameter space one might encounter intermediate shocks, which play such a prominent role in all evolutionarity argumentations.

Recently, Goedbloed (2008) classified the MHD shocks by rewriting the RH equations in the de Hoffmann-Teller frame (de Hoffmann \& Teller (1950)) introducing the existence of a distinct time reversal duality between entropy-allowed and entropy-forbidden solutions. This work encouraged us to revisit the classical RH conditions and augment these results in terms of the commonly exploited shock parameters in the shock frame. Therefore, another goal in this paper is to determine in which regions of parameter space slow, intermediate and fast shocks can appear and how this relates to Goedbloed's analysis, in particular regarding the duality between solutions.

\subsection{The Rankine-Hugoniot jump conditions}

The ideal MHD equations are a system of partial differential equations. When allowing for large amplitude waves, which in the limit case become discontinuous, these equations are replaced by their (weak) discontinuous form: the RH relations. These RH relations express jump conditions across the discontinuity. In any frame where the shock is stationary, the MHD RH conditions become

$$
\left[\left[\begin{array}{c}
\rho v_{n} \\
\rho v_{n}^{2}+p+\frac{B_{t}^{2}}{2} \\
\rho v_{n} v_{t}-B_{n} B_{t} \\
v_{n}\left(\frac{\gamma}{\gamma-1} p+\rho \frac{v_{n}^{2}+v_{t}^{2}}{2}+B_{n}^{2}\right)-B_{n} B_{t} v_{t} \\
v_{n} B_{t}-v_{t} B_{n} \\
B_{n}
\end{array}\right]\right]=\mathbf{0},
$$


where $[[G]]$ expresses the jump $G_{2}-G_{1}$ across the shock. In this paper, index 2 refers to the downstream state, and index 1 refers to the upstream state, while index $n$ refers to the direction of the shock normal, and index $t$ refers to the tangential vector components in the plane spanned up by $\mathbf{B}_{1}$ and $\mathbf{B}_{2}$. Further, $\rho$ is the mass density, $\mathbf{v}$ is the velocity, $p$ the thermal pressure and $\mathbf{B}$ the magnetic field. The ratio of specific heats, $\gamma$, is considered a constant parameter, as we will assume an ideal gas equation of state. For a derivation of these well-known expressions, we refer to de Hoffmann \& Teller (1950), Jeffrey \& Taniuti (1964), Liberman \& Velikhovich (1986), Goedbloed \& Poedts (2004). The eight governing MHD equations have been reduced to six jump conditions in equation (1.1). Three equations have been dropped from the fact that tangential magnetic field components are forced to lie in the same plane perpendicular to the shock front itself: the conservation of momentum reduced to two equations and Faraday's law reduces to a single equation. On the other hand, in the stationary case, the $\nabla \cdot \mathbf{B}=0$ constraint becomes a full equation and is added to equation (1.1).

These six jump conditions can be further restricted by noting that $B_{n}$ is invariant across the shock. Since the RH conditions are translation invariant, the exact values of $v_{t, 1}$ and $v_{t, 2}$ can be eliminated for $\left[\left[v_{t}\right]\right]$, which is completely determined by $\left[\left[B_{t}\right]\right]$. Therefore only four quantities really matter. Hence we now suppose that one state, $\mathbf{u}_{k}=\left(\rho, v_{n}, p, B_{t}\right)_{k}$ is known, where from now on we consistently drop the subscript $k$ from known state quantities. We want to express the other unknown state, $\mathbf{u}_{u}$, in function of this known state. In order to do so, we introduce three dimensionless parameters connected to the known state. First, the plasma- $\beta$, which expresses the ratio of thermal and magnetic pressure. Then, the tangent $\theta$ of the angle between the shock normal and the magnetic field in the known state. Finally the Alfvén Mach number $M$, which is the ratio of the normal velocity component in the known state and the normal Alfvén speed in that region, thus

$$
\begin{aligned}
\beta & \equiv \frac{2 p}{B_{n}^{2}+B_{t}^{2}}, \\
\theta & \equiv \frac{B_{t}}{B_{n}}, \\
M & \equiv \frac{\left|v_{n}\right|}{a_{n}} .
\end{aligned}
$$

We introduced the normal Alfvén speed $a_{n} \equiv \sqrt{\frac{B_{n}^{2}}{\rho}}$. Analogously, we define the tangential Alfvén speed $a_{t} \equiv \sqrt{\frac{B_{t}^{2}}{\rho}}$ and the total Alfvén speed $a \equiv \sqrt{a_{n}^{2}+a_{t}^{2}}$. The full set of characteristic speeds of the full set of MHD equations is given by $\left\{v_{n} \pm v_{f}, v_{n} \pm a_{n}, v_{n} \pm v_{s}, v_{n}\right\}$, where the fast speed $v_{f}$ and the slow speed $v_{s}$ are defined as

$$
\begin{aligned}
& v_{f}^{2} \equiv \frac{1}{2}\left(c^{2}+a^{2}+\sqrt{\left(c^{2}+a^{2}\right)^{2}-4 c^{2} a_{n}^{2}}\right), \\
& v_{s}^{2} \equiv \frac{1}{2}\left(c^{2}+a^{2}-\sqrt{\left(c^{2}+a^{2}\right)^{2}-4 c^{2} a_{n}^{2}}\right),
\end{aligned}
$$

and where we introduced the sound speed $c \equiv \sqrt{\frac{\gamma p}{\rho}}$. On the other hand, the restricted set of planar MHD equations, describing MHD dynamics with all vector quantities restricted to lie in the same plane, has only five characteristic speeds 
$\left\{v_{n} \pm v_{f}, v_{n} \pm v_{s}, v_{n}\right\}$. Ignoring cold MHD, where the thermal pressure $p$ vanishes, and assuming that the normal component of the magnetic field $B_{n}$ (which can be seen as an equation parameter now) does not vanish, we conclude that the planar system is strictly hyperbolic, since $v_{f}=v_{s}$ would imply that both $a$ and $c$ would vanish. Obviously $c$ cannot vanish, and for the same reason also $v_{s} \neq 0$.

\subsection{MHD shock types: classical $1-2-3-4$ classification}

Since the RH conditions do not necessarily lead to a unique solution, it is common to introduce another discrete characterisation: the shock type $i \rightarrow j$. It is well-known (Liberman \& Velikhovich (1986)) that once the shock type and one state is given, if a solution exists, it must be unique. Since $0 \leqslant v_{s} \leqslant a_{n} \leqslant v_{f}$, the full set of MHD equations is hyperbolic, but non-strictly hyperbolic. Those characteristic speeds categorize the up- and downstream states into four types (and three transition types plus a stationary type for completeness), which in order of increasing entropy $S \equiv \frac{p}{\rho^{\gamma}}$ are

- (1) superfast: $v_{f}<\left|v_{n}\right|$,

- (1=2) fast: $\left|v_{n}\right|=v_{f}$,

- (2) subfast: $a_{n}<\left|v_{n}\right|<v_{f}$,

- (2=3) Alfvén: $a_{n}=\left|v_{n}\right|$,

- (3) superslow: $v_{s}<\left|v_{n}\right|<a_{n}$,

- (3=4) slow: $\left|v_{n}\right|=v_{s}$,

- (4) subslow: $\left|v_{n}\right|<v_{s}$,

- $(\infty)$ static: $v_{n}=0$.

We call a shock admissible if it satisfies the second law of thermodynamics: $[[S]]>0$ and admissible versus inadmissible shocks can be related through the time duality principle from Goedbloed (2008). When the upstream state is of type $i$ and the downstream state is of type $j$, then the shock type is $i \rightarrow j$. Furthermore, in terms of these shock types, the admissibility condition translates as $i<j$. The $\mathrm{RH}$ conditions (1.1) together with the admissibility condition $(i<j)$ now allow for the following types of shocks:

- $1 \rightarrow 2$-shocks are called fast shocks. They satisfy $\theta_{2}>\theta_{1}>0$ or $\theta_{2}<\theta_{1}<0$, i.e. the magnetic field gets refracted away from the shock normal.

- $3 \rightarrow 4$-shocks are called slow shocks. They satisfy $\theta_{1}>\theta_{2}>0$ or $\theta_{1}<\theta_{2}<0$, i.e. the magnetic field gets refracted towards the shock normal.

- $1 \rightarrow 2=3$-shocks are called switch-on shocks, since they satisfy $\theta_{1}=0$, i.e. the upstream magnetic field is aligned with the shock normal.

- $2=3 \rightarrow 4$-shocks are called switch-off shocks, since they satisfy $\theta_{2}=0$, i.e. the downstream magnetic field is aligned with the shock normal.

- $1 \rightarrow 3,1 \rightarrow 4,2 \rightarrow 3$ and $2 \rightarrow 4$-shocks are called intermediate shocks. They satisfy $\theta_{2}>0>\theta_{1}$ or $\theta_{2}<0<\theta_{1}$, such that the tangential field flips across the shock normal.

- $2=3 \rightarrow 2=3$-discontinuities are called Alfvén discontinuities. They satisfy $\theta_{1}+\theta_{2}=0$, such that the upstream and the downstream state are equal, except for a sign change of $B_{t}$.

- $\infty \rightarrow \infty$-discontinuities are called contact discontinuities, when only $\rho$ jumps across them. When $B_{n} \equiv B_{n, 1}=B_{n, 2}$ vanishes, $\theta_{1}$ and $\theta_{2}$ are not well-defined, and we call these discontinuities tangential discontinuities.

The latter two cases are called linear discontinuities, since $\left[\left[v_{n}\right]\right]$ vanishes. The other cases are called MHD shocks. All these different shock types are shown in figure 1. 


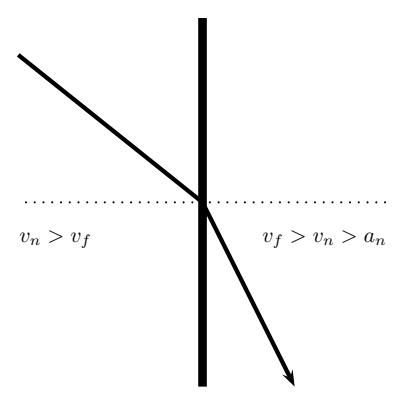

(a)

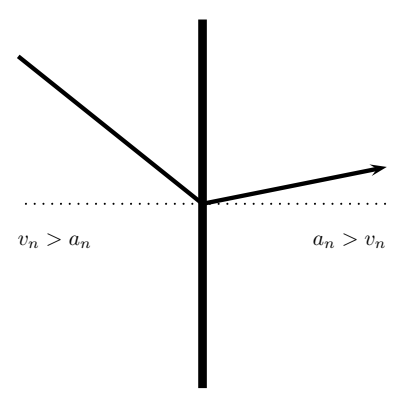

(c)

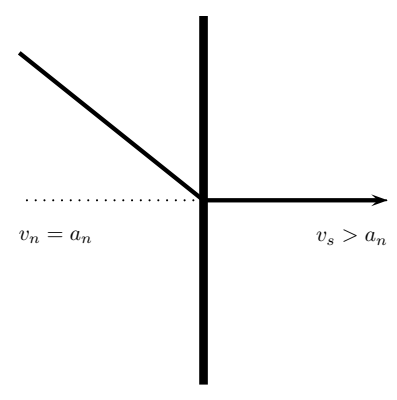

(e)

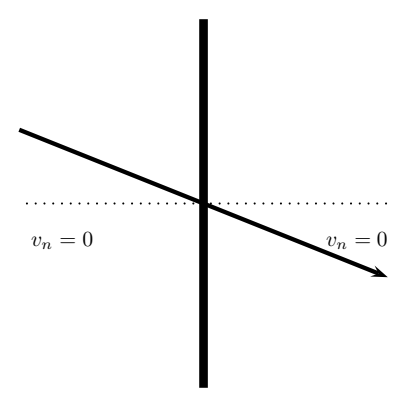

(g)

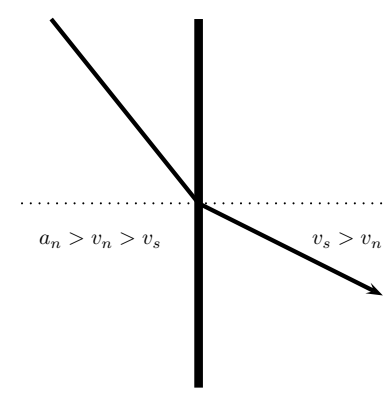

(b)

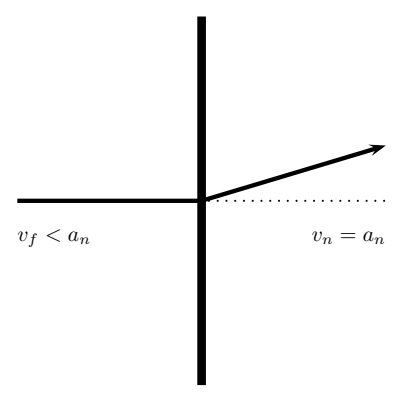

(d)

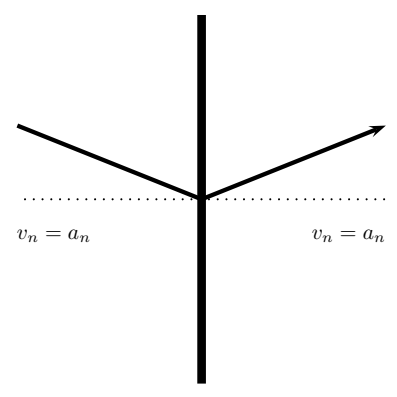

(f)

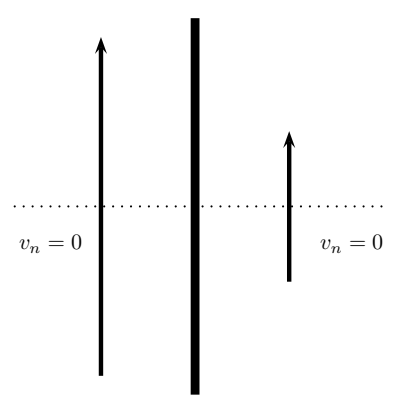

(h)

Figure 1: The classical $1-2-3-4$ classification of MHD discontinuities: a) fast shock; b) slow shock; c) intermediate shock; d) switch-on shock; e) switch-off shock; f)Alfvén discontinuity; g) contact discontinuity; h) tangential discontinuity. 
The classification given above is well-known and features in many classical textbooks, such as Liberman \& Velikhovich (1986). Recently, a contribution to this classical theory was described by Goedbloed (2008), where the RH conditions for MHD shocks were rewritten in an insightful, symmetric form by changing to the de Hoffmann-Teller frame, where $\left[\left[v_{t}\right]\right]=\left[\left[B_{t}\right]\right]$ (see de Hoffmann \& Teller (1950)). In that work, the central role played by intermediate shocks was emphasized, and time reversal duality arguments were introduced, linking admissible and inadmissible shocks. Here we augment this recent study by paying attention to how the MHD shocks appear in the shock reference frame, and analyzing under which conditions intermediate shocks occur.

\section{Solution to the Rankine-Hugoniot conditions}

We scale densities to the known density $\rho$ and magnetic fields to $B_{n}\left(B_{n}\right.$ is constant and equal for known and unknown state, as seen in equation (1.1)). Since no distances or times are involved, the problem is now uniquely scaled. Note that velocities are scaled to the known normal Alfvén speed $a_{n}$. The known state is now given by

$$
\mathbf{u}_{k} \equiv\left(\rho, v_{n}, p, B_{t}\right)=\left(1, \sigma M, \frac{\beta\left(1+\theta^{2}\right)}{2}, \theta\right),
$$

where $\sigma \equiv-1$ when the known state is upstream and $\sigma \equiv 1$ when the known state is downstream, and $\sigma$ just gives the proper sign to the known normal velocity component.

Under the assumption that $M \neq 1$ and $\theta \neq 0$, solving the $\mathrm{RH}$ equations leads to the unknown state quantities

$$
\begin{aligned}
\rho_{u} & =\frac{M^{2} \psi}{\theta\left(M^{2}-1\right)+\psi}, \\
v_{n, u} & =\sigma \frac{\theta\left(M^{2}-1\right)+\psi}{M \psi} \\
p_{u} & =\left(\frac{2}{\gamma+1}+\frac{\psi^{2}+\theta \psi+2}{M^{2}-1}\right) M^{2}-\frac{\gamma-1}{\gamma+1} \frac{\beta\left(1+\theta^{2}\right)+(\psi-\theta)^{2}}{2}, \\
B_{t, u} & =\psi
\end{aligned}
$$

where $\psi$ satisfies the cubic equation

$$
C(\psi) \equiv \psi^{3}+\tau_{2} \psi^{2}+\tau_{1} \psi+\tau_{0}=0,
$$

and its coefficients are given by

$$
\begin{aligned}
\tau_{2} & =-\theta\left((\gamma-1)\left(M^{2}-1\right)-M^{2}\right), \\
\tau_{1} & =\left(M^{2}-1\right)\left((\gamma-1)\left(M^{2}-1\right)+\gamma\left(\beta\left(\theta^{2}+1\right)+\theta^{2}\right)-2\right), \\
\tau_{0} & =-(\gamma+1) \theta\left(M^{2}-1\right)^{2} .
\end{aligned}
$$

The tangential velocity is then found from

$$
\left[\left[v_{t}\right]\right]=\frac{\theta-\psi}{M} .
$$

We can now define the dimensionless parameters refering to the unknown state 
in function of those in the known state. In our first view, we will keep $\psi$ in the expressions, noting that the cubic equation (2.6) can be seen as a continuously partially differentiable function of the known dimensionless parameters and $\psi$, thus $C(M, \theta, \beta, \psi)$, such that the implicit function theorem ensures that locally $\psi$ can be seen as a $\psi(M, \theta, \beta)$. Moreover $\psi(M, \theta, \beta)$ is continuously partially differentiable whenever $\frac{\partial C}{\partial \psi}(M, \theta, \beta, \psi(M, \theta, \beta))$ does not vanish. This latter restriction means that $\psi$ cannot be a double root of the cubic. As we will show later, this condition is equivalent to the condition that none of the characteristic speeds in the unknown region vanishes.

After straightforward algebra, the expressions for $\left(M_{u}, \theta_{u}, \beta_{u}\right)$ now become

$$
\begin{aligned}
M_{u} & =\sqrt{\frac{\left(M^{2}-1\right) \theta+\psi}{\psi}}, \\
\theta_{u} & =\psi \\
\beta_{u} & =\frac{\left((\gamma-1)\left((\theta-\psi)^{2}+\left(1+\theta^{2}\right) \beta\right)-4 M^{2}\right)\left(M^{2}-1\right)+2 M^{2}(\psi(\theta+\psi))}{\left(M^{2}-1\right)(\gamma+1)\left(1+\psi^{2}\right)}
\end{aligned}
$$

In terms of the dimensionless parameters, these equations lead to the following well-known (see e.g. Goedbloed (2008)) invariants across a shock:

$$
\begin{aligned}
{\left[\left[\left(M^{2}-1\right) \theta\right]\right] } & =0, \\
{\left[\left[2 M^{2}+\beta\left(1+\theta^{2}\right)+\theta^{2}\right]\right] } & =0, \\
{\left[\left[\left(\frac{\gamma}{\gamma-1} \beta+M^{2}\right)\left(1+\theta^{2}\right) M^{2}\right]\right] } & =0 .
\end{aligned}
$$

When we define the new quantity $\Omega$, computed from the coefficients of the governing cubic equation (2.6), as

$$
\Omega \equiv 27 \tau_{0}^{2}+4 \tau_{1}^{3}+4 \tau_{2}^{2} \tau_{0}-\tau_{2}^{2} \tau_{1}^{2}-18 \tau_{2} \tau_{1} \tau_{0},
$$

it is well-known that the cubic $C(\psi)$ has three real solutions when

$$
\Omega<0 .
$$

Mathematically speaking, the RH conditions are thus governed by the existence and multiplicity of the real roots of a cubic, hence $\Omega=0$ will play a crucial role, as will be explained in section 3. It should be noted, however, that most analytical expressions in this choice of frame and parametrization are complicated expressions, e.g. one can note that $\Omega$ is a polynomial of degree 6 in $M^{2}$, where $\left(M^{2}-1\right)$ appears as a double factor. The same governing equations, as expressed in the de HoffmannTeller frame exploited by Goedbloed (2008) give a much more compact algebraic form along with an easier classification.

\section{Physical meaning of $\Omega$}

Note that $\Omega=0$ is exactly the condition for having a real solution with multiplicity two to $C(\psi)=0$. Therefore, whenever $\Omega \neq 0,\left(M_{u}, \theta_{u}, \beta_{u}\right)$ is a smooth function of $(M, \theta, \beta)$. A related observation is that equation (2.18) is the analytical condition for the existence of an intermediate shock, since when only one solution exists to the $\mathrm{RH}$ jump conditions it must be a fast or a slow shock (see e.g. Liberman \& Velikhovich (1986)). 

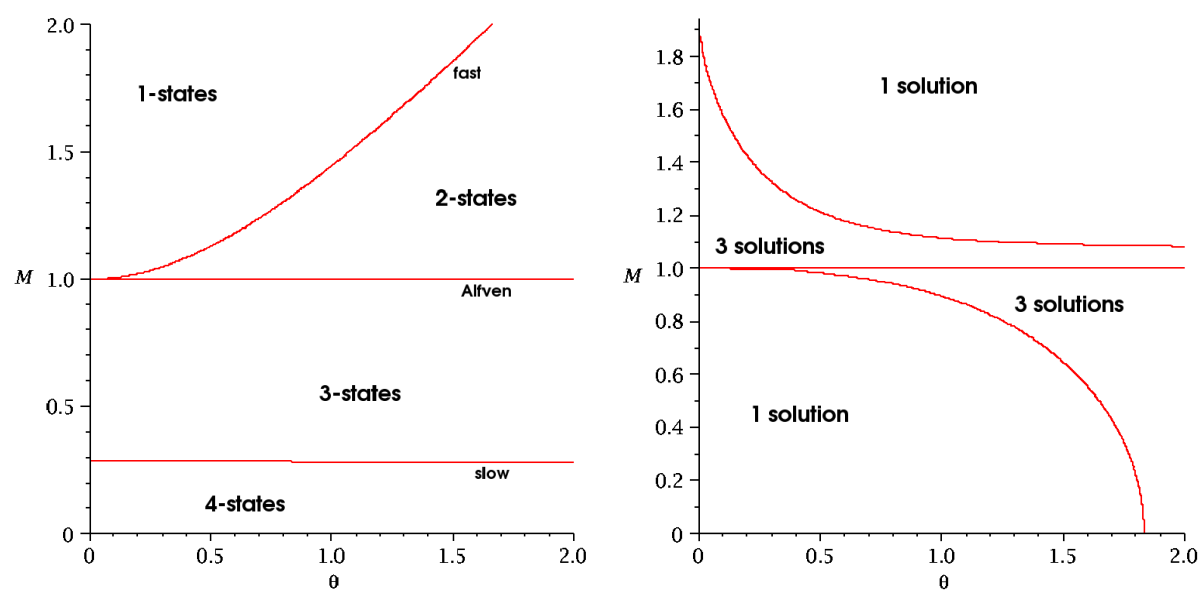

Figure 2: Left: The $(\theta, M)$ state plane for $\beta=\frac{1}{10}$ and $\gamma=\frac{5}{3}$. Shown are the curves fast: $v_{n}=\overline{v_{f}}$, Alfvén: $v_{n}=a_{n}$ and slow: $v_{n}=v_{s}$. These curves separate the $(\theta, M)$ plane in the classical $1-2-3-4$ state regions. Right: Shown is the curve $\Omega=0$. Note that states with $M=1$ appear as a double zero of $\Omega$. Where $\Omega>0$, only one real solution to the $\mathrm{RH}$ conditions exist. On the other hand, where $\Omega<0$, there exist three real solutions to the $\mathrm{RH}$ conditons. On $\Omega=0$, the $\mathrm{RH}$ solutions allow for 2 distinct real solutions.

We will argue that all the states, which can be connected to a state which is exactly fast, slow or Alfvénic, satisfy $\Omega=0$. In other words: the surface $\omega \equiv$ $\{(M, \theta, \beta) \mid \Omega(M, \theta, \beta)=0\}$ in our 3D parameter space will be shown to correspond to all states which can be connected by the RH conditions to known states satisfying $\left(v_{n}-a_{n}\right)\left(v_{n}^{2}-v_{f}^{2}\right)\left(v_{n}^{2}-v_{s}^{2}\right)=0$. One can visualize these concepts by drawing the corresponding curves in the $(\theta, M)$-plane, for fixed $\beta$ and $\gamma$. This is done in figure (2), where the plot at left concentrates on the $1-2-3-4$ state regions, and the panel at right shows $\Omega=0$ curves.

First, we make some general observations. 1-states can be connected to a 2-state, a 3-state and a 4-state. Therefore, they can only appear as an upstream state. There are at most three different real solutions with a 1-state as an known upstream state. When there is only one solution, this solution is a fast $1 \rightarrow 2$ shock. In the transition case where two different real solutions exist, these solutions include one fast $(1 \rightarrow 2)$ shock, and two coinciding intermediate $1 \rightarrow 3=4$ shocks. Finally, when there are three different real solutions, one of these is a fast $(1 \rightarrow 2)$ shock and the 2 other solutions are respectively an intermediate $1 \rightarrow 3$ and an intermediate $1 \rightarrow 4$ shock.

Similarly, 2-states can be connected to a 1-state, a 3 -state and a 4 -state. Therefore, the admissibility condition which demands that $i<j$ for an $i \rightarrow j$ shock, ensures that a 2-state can, at most, occur in one downstream state solution and two upstream state solutions to the RH conditions. Again, when only one real solution exists, it is a fast $(1 \rightarrow 2)$ shock. In the transition case where two different real solutions exist, these solutions include one fast shock and two coinciding intermediate $2 \rightarrow 3=4$ shocks. Finally, when there are three different real solutions, one of these is a fast shock, and the 2 other solutions are respectively an intermediate $2 \rightarrow 3$ and an intermediate $2 \rightarrow 4$ shock.

The same reasoning states that generally speaking, 3-states can be connected to 
a 1-state, a 2-state and a 4-state. The admissibility condition ensures that they can, at most, occur in two downstream state solutions and one upstream state solution to the RH conditions. Now, when only one real solution exists, it is a slow $(3 \rightarrow 4)$ shock. In the transition case where two different real solutions exist, these solutions include one slow $(3 \rightarrow 4)$ shock and two coinciding intermediate $1=2 \rightarrow 3$ shocks. Finally, when there are three different real solutions, one of these is a slow shock, and the 2 other solutions are respectively an intermediate $1 \rightarrow 3$ and an intermediate $2 \rightarrow 3$ shock.

Generally speaking, 4-states can be connected to a 1-state, a 2-state and a 3state. Therefore, the admissibility condition ensures that they can, at most, occur in three downstream state solutions to the $\mathrm{RH}$ conditions. Again, when only one real solution exists, it is a slow $(3 \rightarrow 4)$ shock. In the transition case where two different real solutions exist, these solutions include one slow $(3 \rightarrow 4)$ shock and two coinciding intermediate $1=2 \rightarrow 4$ shocks. Finally, when there are three different real solutions, one of these is a slow $(3 \rightarrow 4)$ shock and the 2 other solutions are respectively an intermediate $1 \rightarrow 4$ and an intermediate $2 \rightarrow 4$ shock.

These general observations now allow us to consider the transition cases. A $1=2$ state, where $v_{n}=v_{f}$ or a $3=4$-state, where $v_{n}=v_{s}$, will always have the trivial solution. Therefore they can only appear in none or two real non-trivial intermediate solutions, depending on the sign of $\Omega$. Both these solutions, labeled as unknown state $a$ and $b$, have $\mathbf{u}_{k}$ as a double solution, thereby satisfying $\Omega_{u, a}=\Omega_{u, b}=0$, and as we will show in section 4.2 mutually satisfy the $\mathrm{RH}$ conditions. This is argued in what follows.

Consider an upstream state which satisfies $v_{n}=v_{f}$. Straightforward algebra shows that $C(\theta)=0$, so one of the solutions to the $\mathrm{RH}$ jump conditions is the trivial one. By solving $\frac{C(\psi)}{\psi-\theta}=0$, one finds 2 solutions. Those solutions are real only when $\Omega \leqslant 0$. In this case they lead to a $1=2 \rightarrow 3$ and a $1=2 \rightarrow 4$ solution, which we respectively call $\mathbf{u}_{u, a}$ and $\mathbf{u}_{u, b}$. These $\mathbf{u}_{u, a}$ and $\mathbf{u}_{u, b}$ mutually satisfy the $\mathrm{RH}$ conditions and can therefore be seperated by a slow $3 \rightarrow 4$ shock. Both these solutions have our specific known upstream state $\mathbf{u}_{k}$, where $v_{n}=v_{f}$, as a double solution, and therefore satisfy $\Omega_{u, a}=\Omega_{u, b}=0$.

Completely analogously, consider now a downstream state with $v_{n}=v_{s}$. Straightforward algebra shows that $C(\theta)=0$, so one of the solutions to the RH jump conditions is the trivial one. By solving $\frac{C(\psi)}{\psi-\theta}=0$, one finds 2 solutions. Those solutions are real only when $\Omega \leqslant 0$. In this case they lead to a $1 \rightarrow 3=4$ and a $2 \rightarrow 3=4$ solution, which we respectively call $\mathbf{u}_{u, a}$ and $\mathbf{u}_{u, b}$. We again find that $\mathbf{u}_{u, a}$ and $\mathbf{u}_{u, b}$ mutually satisfy the RH conditions and can therefore be separated by a fast shock. Both these solutions have our specific known downstream state $\mathbf{u}_{k}$, where $v_{n}=v_{s}$, as a double solution, and therefore satisfy $\Omega_{u, a}=\Omega_{u, b}=0$.

Finally, consider a state where $v_{n}=a_{n}$. Now $C(\psi)$ simplifies as $\psi^{3}+\theta_{i} \psi^{2}=0$, where $i=1,2$ selects respectively the up- or the downstream solution. The solutions to the $\mathrm{RH}$ conditions are now a switch-on shock, a switch-off shock and a rotational wave. The switch-on and the switch-off solution also mutually satisfy the RH jump condition. This can be easily checked by straightforward algebra. Note that in this case $\Omega_{u}=0$ automatically. 


\section{Results}

Since the governing expressions in the shock frame are complicated, all calculations reported here are performed by a symbolic computational software package, namely MAPLE 11.0. We search for critical values for the parameters in both up- and downstream states, for which different shock types can occur. These critical values are found by simple geometrical arguments using the knowledge obtained so far: namely that in the $(\theta, M)$-plane, 2 families of curves as shown in figure (2) split up the state plane in various regions. Unless explicitly stated otherwise, we assume from now on that $\gamma=\frac{5}{3}$.

\section{1. $(\theta, M)$-diagrams at fixed $\beta$}

For varying $\beta$, we plot both the curves where the known upstream normal velocity component $v_{n}$ is exactly fast, Alfvénic or slow (i.e. $v_{n}=v_{f}, v_{n}=a_{n}, v_{n}=v_{s}$ ) and the curves $\omega: \Omega=0$ in a $(\theta, M)$-diagram in figure (3). Those curves divide the $(\theta, M)$-parameter space in regions, where certain types of shocks are mathematically possible. An important transition occurs at $\beta=\frac{2}{\gamma}$. Here the sound speed equals the Alfvén speed and the thermal pressure equals the magnetic pressure. When $\beta<\frac{2}{\gamma}$, we call the plasma magnetically dominated. In this case, the curves $v_{n}=v_{f}$ and $v_{n}=a_{n}$ have a common point at $(\theta, M)=(0,1)$. When the plasma is thermally dominated, $\beta>\frac{2}{\gamma}$, the curves $v_{n}=v_{s}$ and $v_{n}=a_{n}$ have a common point at $(\theta, M)=(0,1)$.

Every region is then coded with a latin number code for $(\theta, M)$, indicative of an upstream state, i.e. $\left(\theta_{1}, M_{1}\right)$. This means:

- (I) One fast shock and two intermediate shocks of type $1 \rightarrow 3$ and $1 \rightarrow 4$ are possible;

- (II) Only a fast shock is possible;

- (III) Two intermediate shocks of type $2 \rightarrow 3$ and $2 \rightarrow 4$ are possible;

- (IV) No shocks are possible;

- (V) Only a slow shock is possible.

We can interpret the graphs in terms of the downstream state in a similar manner, where $(\theta, M)=\left(\theta_{2}, M_{2}\right)$. Every region is also coded with a letter code, meaning the following:

- (A) One slow shock and two intermediate shocks of type $2 \rightarrow 4$ and $3 \rightarrow 4$ are possible;

- (B) Only a slow shock is possible;

- (C) Two intermediate shocks of type $1 \rightarrow 4$ and $2 \rightarrow 4$ are possible;

- (D) No shocks are possible;

- (E) Only a fast shock is possible.

These regions are shown in figure (3), where the axes labels must be interpreted as $\left(\theta_{2}, M_{2}\right)$ instead of $\left(\theta_{1}, M_{1}\right)$ from above.

The $1-2-3-4$ classification divides the $(\beta, M, \theta)$-space into four regions. The surface $\omega: \Omega=0$ divides each of those regions into two: one region where $\Omega>0$ and one where $\Omega<0$, such that the $(\beta, M, \theta)$ space is divided into eight regions by these curves.

The time reversal duality principle from Goedbloed (2008) is hereby made visible in the fact that every region corresponds to 1 or 3 mathematical solutions, but the coding tells whether the state can appear as an upstream or as a downstream region. 


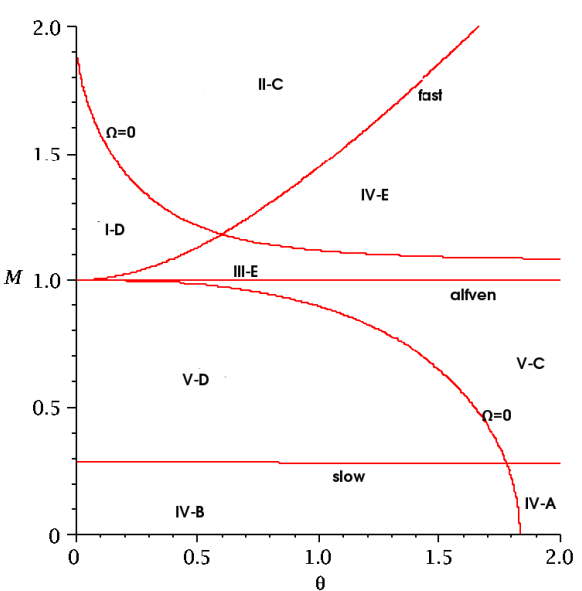

(a)

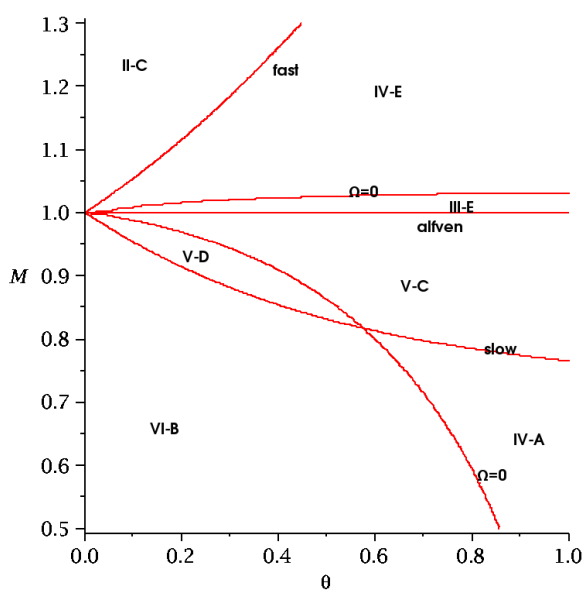

(c)

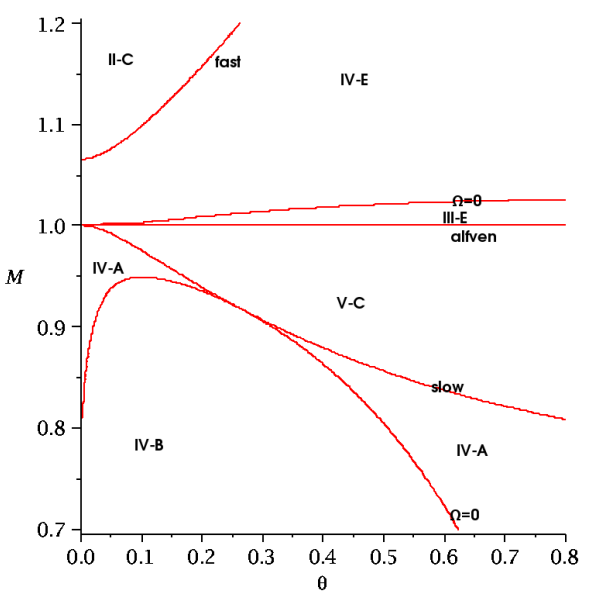

(e)

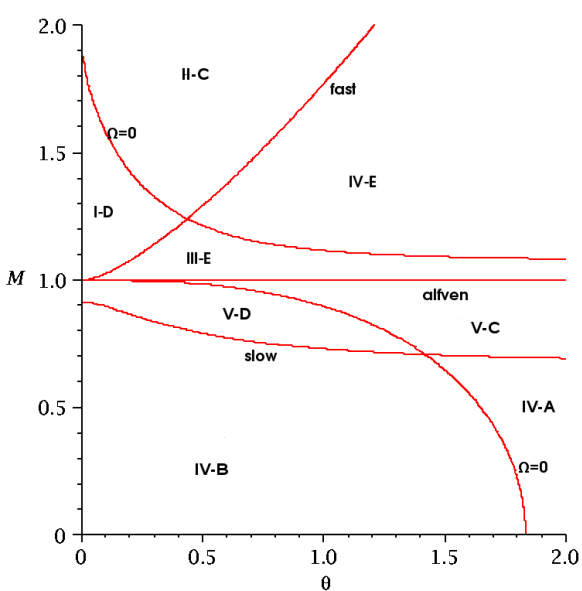

(b)

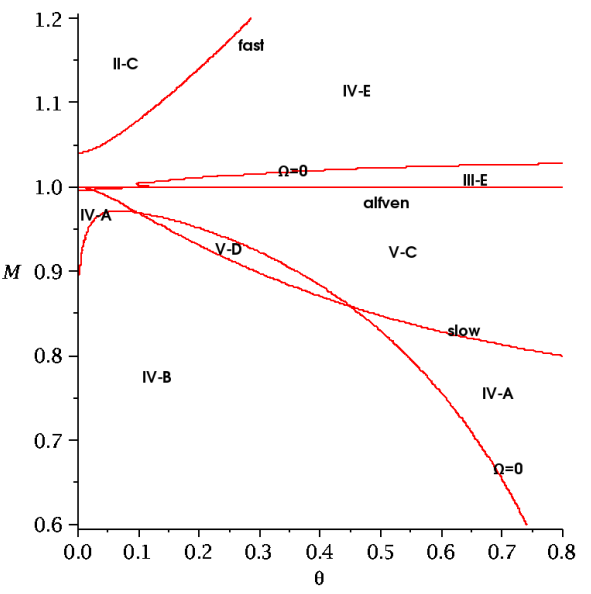

(d)

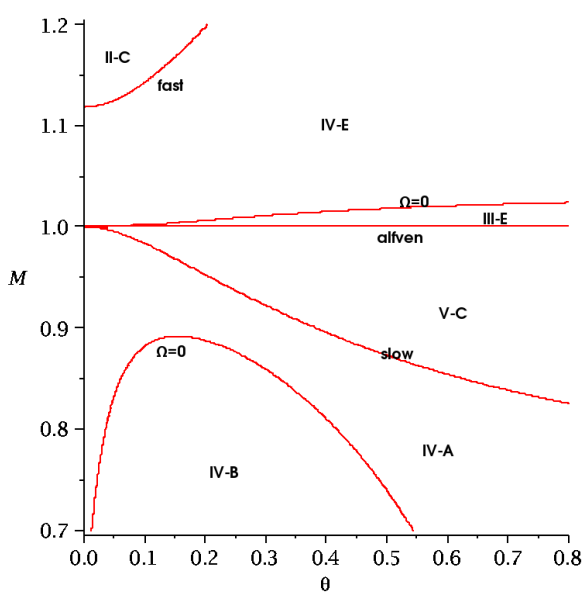

(f)

Figure 3: Parameter space divided into various regions. Panels differ in their $\beta$ value: a) $\beta=0.1$, b) $\beta=1.0$, c) $\beta=1.2=\frac{2}{\gamma}$, d) $\beta=1.3$, e) $\beta=1.3637$, f) $\beta=1.5$. The latin cypher-letter code is explained in the text, and related to the time reversal duality argument put forth by Goedbloed (2008). 


\subsection{Equivalence classes introduced by the $R H$ conditions}

The RH conditions are equivalent to equations (2.14-2.16), which express the existence of three shock invariants. Therefore two states can be connected through the stationary RH conditions if and only if they have the same value for the expression $\zeta_{1} \equiv\left(M^{2}-1\right) \theta, \zeta_{2} \equiv 2 M^{2}+\beta\left(1+\theta^{2}\right)+\theta^{2}$ and $\zeta_{3} \equiv\left(\frac{\gamma}{\gamma-1} \beta+M^{2}\right)\left(1+\theta^{2}\right) M^{2}$. Denoting the relation "state $A$ can be connected to state $B$ through the stationary $\mathrm{RH}$ conditions" as $A \mapsto_{\mathrm{RH}} B$, this relation $\mapsto_{\mathrm{RH}}$ is an equivalence. Indeed:

- $\mapsto \mathrm{RH}$ is reflexive: $A \mapsto \mathrm{RH} A$. Every state can be connected to itself through the stationary $\mathrm{RH}$ conditions.

- $\mapsto \mathrm{RH}$ is symmetric: $A \mapsto \mathrm{RH} B \Rightarrow B \mapsto \mathrm{RH} A$. If state $A$ can be connected to state $B$ through the stationary RH conditions, then also state $B$ can be connected to state $A$ by these conditions. Of course only one of these connections satisfies the entropy condition.

- $\mapsto \mathrm{RH}$ is transitive: $A \mapsto_{\mathrm{RH}} B \wedge B \mapsto_{\mathrm{RH}} C \Rightarrow A \mapsto_{\mathrm{RH}} C$. Indeed: if $A \mapsto_{\mathrm{RH}} B$, then $\zeta_{i}(A)=\zeta_{i}(B)$, and if $B \mapsto \mathrm{RH} C$, then $\zeta_{i}(B)=\zeta_{i}(C)$. Hence $A \mapsto \mathrm{RH} B \wedge B \mapsto \mathrm{RH}$ $C$ implies $\zeta_{i}(A)=\zeta_{i}(B)=\zeta_{i}(C)$, which means that $A \mapsto \mathrm{RH} C$.

We conclude that $\left(\zeta_{1}, \zeta_{2}, \zeta_{3}\right)$ defines equivalence classes on the parameter space. All these equivalence classes contain one, two, three or four states.

A state $A$ is in an equivalence class with one element in the following cases:

- If $M=1$ and $\theta=0$. In this degenerate case the switch-on solution, the switch off-solution and the rotational solution all coincide.

- If $\Omega>0$, and $\left(v_{n}^{2}-v_{s}^{2}\right)\left(v_{n}^{2}-v_{f}^{2}\right)=0$. In this case, the only real solution to the RH conditions is $A$ itself.

A state $A$ is in an equivalence class with two elements, in the following cases.

- If $\Omega>0$ and $\left(v_{n}^{2}-v_{s}^{2}\right)\left(v_{n}^{2}-v_{f}^{2}\right) \neq 0$. In this case the equivalence class contains $A$ itself, and the state introduced by the unique (non-trivial) real solution to the cubic equation (2.6).

- If $\Omega=0$ and $\left(v_{n}^{2}-v_{s}^{2}\right)\left(v_{n}^{2}-v_{f}^{2}\right)=0$. Since $\Omega=0$, the solutions to the $\mathrm{RH}$ conditions crossing the Alfvén speed are coinciding. Since $v_{n}$ is exactly fast or exactly slow, it appears itself as a solution to the cubic.

A state $A$ is in an equivalence class with three elements in the following cases.

- if $\Omega=0$, but $\left(v_{n}^{2}-v_{s}^{2}\right)\left(v_{n}^{2}-v_{f}^{2}\right) \neq 0$. As explained earlier, in this case the $\mathrm{RH}$ conditions have an exactly slow or fast state as a double solution, and a single solution which also satisfies $\Omega=0$.

- if $\Omega<0$ and $v_{n}=v_{f}$ or $v_{n}=v_{s}$. As explained earlier, in this case the RH conditions have 2 different solutions with $\Omega=0$, and the considered state itself as a third solution.

A state is in an equivalence class with four elements in the following case:

- if $\Omega<0$ and $v_{n} \neq v_{f}$ and $v_{n} \neq v_{s}$. As explained above, in this case the RH conditions have three different (non-trivial) solutions. Also, the considered state itself is in the equivalence class.

- if $v_{n}=a_{n}$. The RH conditions now have a switch-on shock, a switch-off shock and a rotational wave as a solution. The considered state itself completes the equivalence class.

Hence, when $A$ is a superfast state, with $\Omega>0$, it is in an equivalence class with exactly one other state. This state should be subfast, since we know that if only one solution exists, it does not cross the Alfvén speed. Also, the solution state has 
only one solution, therefore it also satisfies $\Omega>0$. Interpreting this result on figure (3), we conclude that the $I I-C$-region can only be connected to the $I V-E$-region and the other way around.

Completely analoguously we find that a subslow state with $\Omega>0$ can only be connected with a superslow state, also satisfying $\Omega>0$. Interpreting this again on figure (3), we conclude that the $I V-B$-region can only be connected to the $V-D$ region, and vice versa. Further, we conclude that for any state in the regions $I-D$, $I I I-E, V-C$ and $I V-A$, there exist states in the regions $I-D, I I I-E, V-C$ and $I V-A$ respectively, which can be mutually connected through the RH jump condition. The initial eight regions of parameter space have thus been divided into two groups of two mutually connectable regions and one group of four mutually connectable regions.

If we do not take into account that $\beta_{u}$ should be positive, these connections are mappings. We know the entropy condition in an $i \rightarrow j$ shock reduces to $j>i$. Thus, when we consider one equivalence class we have four or two states, such that the entropy increases with state type. Therefore, we know that when one of the mathematical solutions has negative pressure, it must be the 1-states. When two of those states have negative pressure, it must be the 1-state and the 2-state, and so on. In the next section, we will additionally consider the physical restriction that all pressures should be positive.

\subsection{Positive pressure requirement}

Until now we have only used the nonlinear relations expressed by $\mathrm{RH}$, as well as the admissibility condition, to count the number of solutions in the $(\theta, M, \beta)$ state space. The admissibility restriction is that $[[S]]>0$, which is satisfied for an $i \rightarrow j$-shock if and only if $j>i$. Further restrictions are that the solution should have positive thermal pressure, $p_{u}>0$ and density $\rho_{u}>0$. The latter restriction is trivially satisfied. In fact, we can only encounter problems when the known state is a downstream state, because when the upstream pressure is positive the upstream entropy is also positive. Hence, the downstream entropy is positive and the downstream pressure too. Therefore, we can expect that for a given 1state, the positive pressure requirement is trivially satisfied. When the known state is a 2-state, we expect to encounter one critical surface dividing the 2-state regions into subregions where the fast $(1 \rightarrow 2)$ shock solution has positive versus negative thermal pressure. When the given state is a 3-state, we expect to encounter two of such critical surfaces. And when the given state is a 4-state we expect to encounter three of such critical surfaces. Therefore, the parameter space is now divided in 16 regions, as summarized in table 1 .

Figure (4) shows the regions in which $p_{u}>0$ for $\beta=0.1$ and $\beta=2$. The figure now plots: $(i)$ the three curves defining $v_{n}=v_{s}, v_{n}=a_{n}$ and $v_{n}=v_{f} ;(i i)$ the curves where $\Omega=0$; and (iii) the lines defining $p_{u}=0$. Finding these regions is straightforward: pick the correct root of the cubic, fill it out in the expression for $p_{u}$ and make it vanish. The governing expressions can even be found analytically. As mentioned above, the addition of these $p_{u}=0$ curves now divides the parameter space into 16 regions, namely:

- (i) This state can occur as the upstream state of a fast shock;

- (ii) This state can occur as the downstream state of a fast shock;

- (iii) This state cannot occur, since the only solution has negative pressure; 

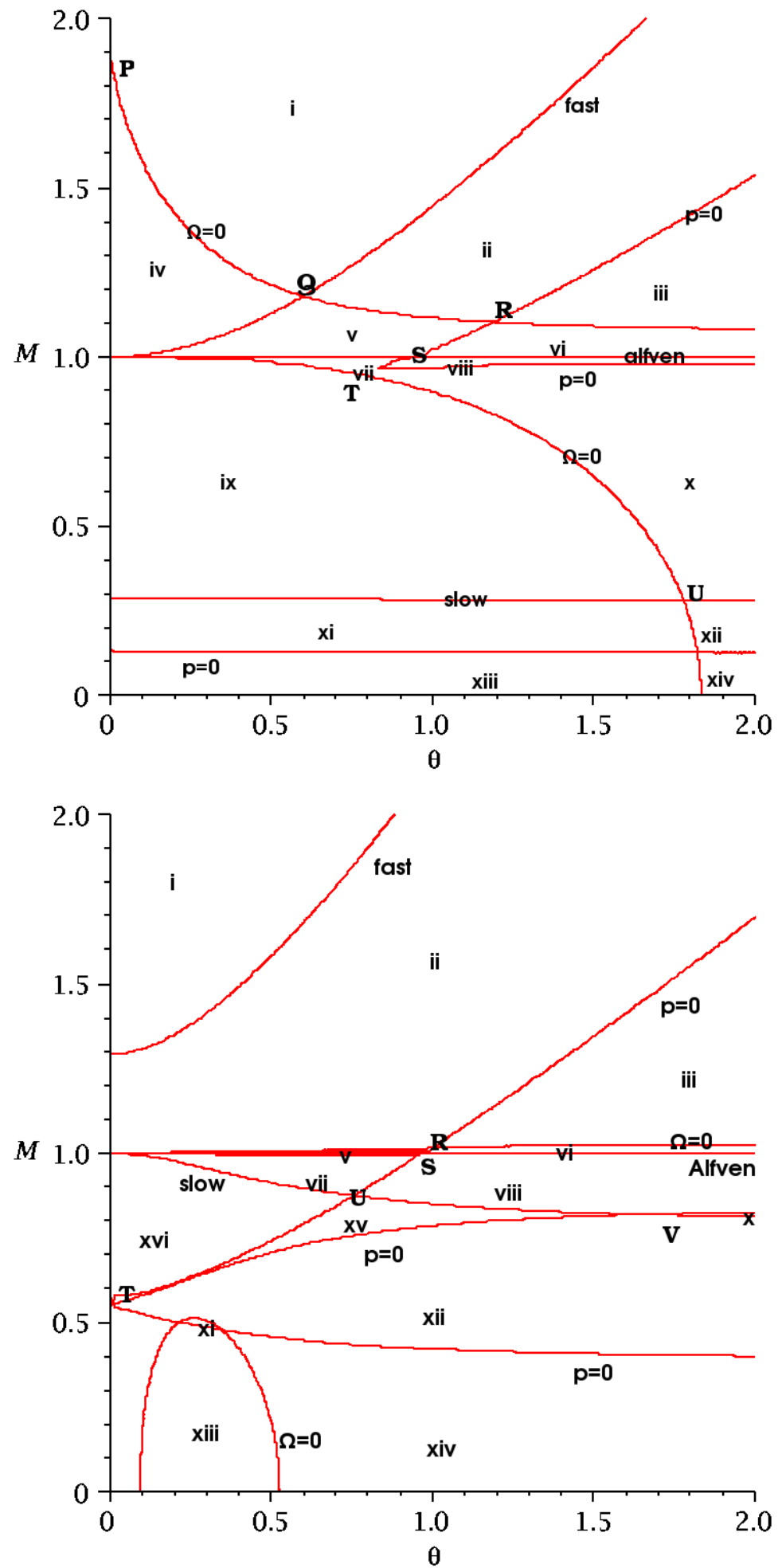

Figure 4: The 16 regions in parameter space where $p_{u}>0$, respectively for $\beta=0.1$ and $\beta=2$. 


\begin{tabular}{|r||c|r|}
\hline & $\Omega<0$ & $\Omega>0$ \\
\hline 1-state & 1 & 1 \\
2-state & 2 & 2 \\
3-state & 3 & 1 \\
4-state & 4 & 2 \\
\hline
\end{tabular}

1: The positive pressure requirement divides the eight regions in parameter space in even more regions. The number of subregions in which the original regions are divided are shown above. Since the region is only important when the given state is downstream, it is not surprising that the total number of regions is doubled from 8 to 16.

- (iv) This state can occur as the upstream state of a fast shock, and as the upstream state of an intermediate $1 \rightarrow 3$ and $1 \rightarrow 4$ shock;

- $(v)$ This state can occur as the upstream state of an intermediate $2 \rightarrow 3$ and $2 \rightarrow 4$ shock, and as the downstream state of a fast shock;

- $(v i)$ This state can occur as the upstream state of an intermediate $2 \rightarrow 3$ and $2 \rightarrow 4$ shock, but not as the downstream state of a fast shock, since the solution would have a negative thermal pressure;

- (vii) This state can occur as the upstream state of a slow shock, or as the downstream state of an intermediate $1 \rightarrow 3$ and $2 \rightarrow 3$ shock;

- (viii) In this region, one of the three solutions has negative pressure. This will always be the $1 \rightarrow 3$-solution. Therefore this region can occur as the upstream state of a slow shock, or as a downstream state of an intermediate $2 \rightarrow 3$ shock;

- $(i x)$ This region can occur as the upstream state of a slow shock;

- $(x)$ This state has two solutions with negative pressure: namely both intermediate solutions. Therefore it can only occur as the upstream state of a slow shock;

- $(x i)$ This state can occur as the downstream region of a slow shock;

- (xii) This state has two solutions with negative pressure: namely both intermediate solutions. Therefore it can only occur as the downstream state of a slow shock;

- (xiii) This state cannot occur, since the single real solution has negative pressure.

- (xiv) This state cannot occur, since all 3 solutions have negative pressure.

- $(x v)$ This state can occur as the downstream state of a slow shock, and as the downstream state of an intermediate $2 \rightarrow 4$ shock, but not as the downstream state of an intermediate $1 \rightarrow 4$ shock, since the solution would have a negative thermal pressure;

- $(x v i)$ This state can occur as the downstream state of a slow shock, and as the downstream state of an intermediate $1 \rightarrow 4$ and $2 \rightarrow 4$ shock;

The combination of graphing the surface defined by $\Omega=0$, together with the surfaces defined by $v_{n}=v_{s}, v_{n}=a_{n}, v_{n}=v_{f}$ and the positive pressure requirement hence provides a complete, but admittedly non-trivial, graphical means to the many possibilities for the MHD shock transitions. We now continue to exploit this knowledge to delimit possible parameter ranges for certain shock types. As a matter of fact, in what follows, we will use figure (4) and how this figure varies with $\beta$, to find limiting values of the parameters at which different shock types can occur. By doing so, we will actually find the equations of the curves traced out in $(\theta, M, \beta)$ parameter space that are labeled with $\mathrm{P}, \mathrm{Q}, \mathrm{R}, \mathrm{S}, \mathrm{T}, \mathrm{U}$ and $\mathrm{V}$ as indicated 
in these plots. These points identify the intersections of the various regions, and thus act to delimit realizable parameter ranges for shocks.

\subsection{Switch-on shocks and switch-off shocks}

Switch-on shocks are possible where $\theta=0$. An extra condition for switch-on shocks to be possible is $\Omega<0$, since otherwise only fast shocks are possible. Note that switch-on shocks are only possible when $\beta<\frac{2}{\gamma}$. In this case the plasma is said to be magnetically dominated. Therefore the maximum value at which we can find switch-on shocks, is found by filling $\theta=0$ out in the expression for $\Omega$. This works out to be

$$
1<M_{\mathbf{S}-\text { on }}<\sqrt{\frac{\gamma(1-\beta)+1}{\gamma-1}} .
$$

For the same reason, switch-off shocks can be found in a completely similar manner, yielding

$$
\sqrt{\frac{\gamma(1-\beta)+1}{\gamma-1}}<M_{\mathrm{S}-\text { off }}<1
$$

whenever $\frac{\gamma(1-\beta)+1}{\gamma-1}>0$, i.e. when $\beta<\frac{\gamma+1}{\gamma}$. This agrees with the well-known expressions for these degenerate shock cases (e.g. Kennel et al. (1989)).

When solving the RH conditions for $\theta=0$, when $\beta<\frac{\gamma+1}{\gamma}$, two switch-on or switch-off solutions exist. For $\gamma=\frac{5}{3}$, these solutions are given by

$$
\begin{aligned}
\beta_{u} & =\frac{1-2 M^{2}-\beta}{(\gamma-1) M^{4}+(\gamma(\beta-2)) M^{2}+\gamma(1-\beta)}-1 \\
M_{u} & =1 \\
\theta_{u} & = \pm \sqrt{\left((\gamma \beta-2)-(\gamma-1)\left(M^{2}-1\right)\right)\left(M^{2}-1\right)} .
\end{aligned}
$$

Finally, there is also another solution, which is a hydrodynamical shock. This solution is given by

$$
\left(\beta_{u}, M_{u}, \theta_{u}\right)=\left(\frac{6 M^{2}-\beta}{4}, \sqrt{\frac{2 M^{2}+5 \beta}{8}}, 0\right),
$$

which only has positive pressure for $\beta<6 M^{2}$.

Figure (5) shows the plane given by $\theta=0$ in parameter space. The graphs plotted are $(i)$ the curves given by $\Omega=0$; (ii) the curve $v_{n}=a_{n}$; (iii) the curve given by $p_{H D} \equiv 6 M^{2}-\beta=0$, which is the limiting curve for the existence of a hydrodynamical shock solution; and (iv) the curve given by $p_{\mathbf{S}-\text { off }} \equiv-4 M^{2}-$ $2 \beta+2+5 M^{2} \beta+2 M^{4}=0$, which is a limiting curve for the existence of a switch-off solution. The entropy condition ensures that there is no equivalent limiting curve for switch-on solutions. Note that the known state of a switch-on shock, where $\theta=0$ is always magnetically dominated, whereas the known state of a switch-off shock, where $\theta=0$ is always thermally dominated. Also shown are the curves where the hydrodynamical shock solutions and the switch-on and switch-off solution have $p_{u}=0$. Again, the intersection point, demarkating different regions, are labeled with $A, B, C$ and $D$ and used in what follows.

We conclude that the maximum Mach number at which switch-on solutions exist, is reached in point $A$, for which $(\theta, M, \beta)=(0,2,0)$, as indicated in figure (5), while the maximum plasma- $\beta$ for switch-on shocks is reached in point $B$, where 


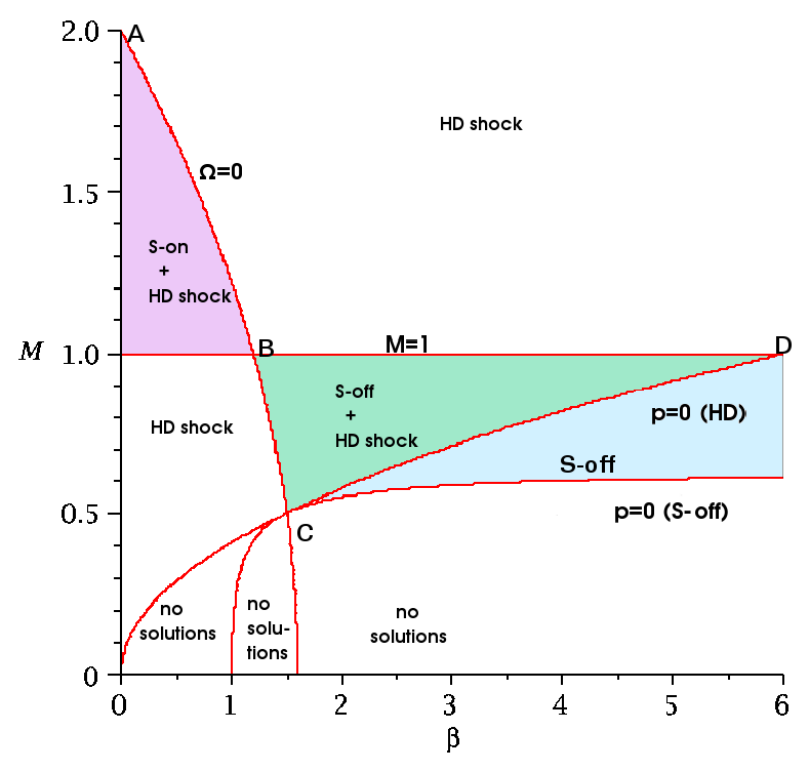

Figure 5: The regions where switch-on or switch-off shocks can occur are colored in greyscale. By finding the coordinates of points $A, B$ and $C$, we know the limiting values for which these shocks can occur. More details are given in the text.

$(\theta, M, \beta)=\left(0,1, \frac{2}{\gamma}=1.2\right)$. Therefore the Mach number for the existence of a switch on-shock is bounded by $1<M<2$ and the plasma- $\beta$ at which these shocks can occur must satisfy $0<\beta<\frac{2}{\gamma}=1$.2. For switch-off shocks, the minimum plasma- $\beta$ is reached in point $B$ and is therefore $\beta=\frac{6}{5}=1.2$. The minimum value of the Mach number $M$ is reached in point $C$ whose coordinates are found by solving

$$
\left\{\begin{array}{ccc}
p_{u} & = & 0 \\
\sqrt{\frac{\gamma(1-\beta)+1}{\gamma-1}} & = & M .
\end{array}\right.
$$

Therefore $C$ satisfies $(\theta, M, \beta)=\left(0, \sqrt{\frac{\gamma-1}{\gamma+1}}, \frac{4}{\gamma+1}\right)=(0,0.5,1.5)$, and switch-off shocks satisfy

$$
\frac{1}{2}=\sqrt{\frac{\gamma-1}{\gamma+1}} \leqslant M_{\mathrm{S}-\text { off }}<1 .
$$

In fact, it is straightforward to show that in $C$, both the curves $p_{H D}=0$ and $p_{\mathbf{S}-\text { off }}=0$ touch, while $\omega: \Omega=0$ also contains $C$.

Regarding HD shocks, the maximum plasma- $\beta$ at which they can exist is reached in $D$, where $(\theta, M, \beta)=\left(0,1, \frac{4}{\gamma-1}\right)=(0,1,6)$, such that all HD shocks satisfy $\beta<6$.

Finally note that $p_{\mathbf{S}-\text { off }}=0$ has $M=\sqrt{\frac{\gamma-1}{\gamma}}=0.6325$ as a horizontal asymptotic, such that for $\theta=0$, the $\mathrm{RH}$ conditions always lead to at least one solution whenever $M>0.6325$. 

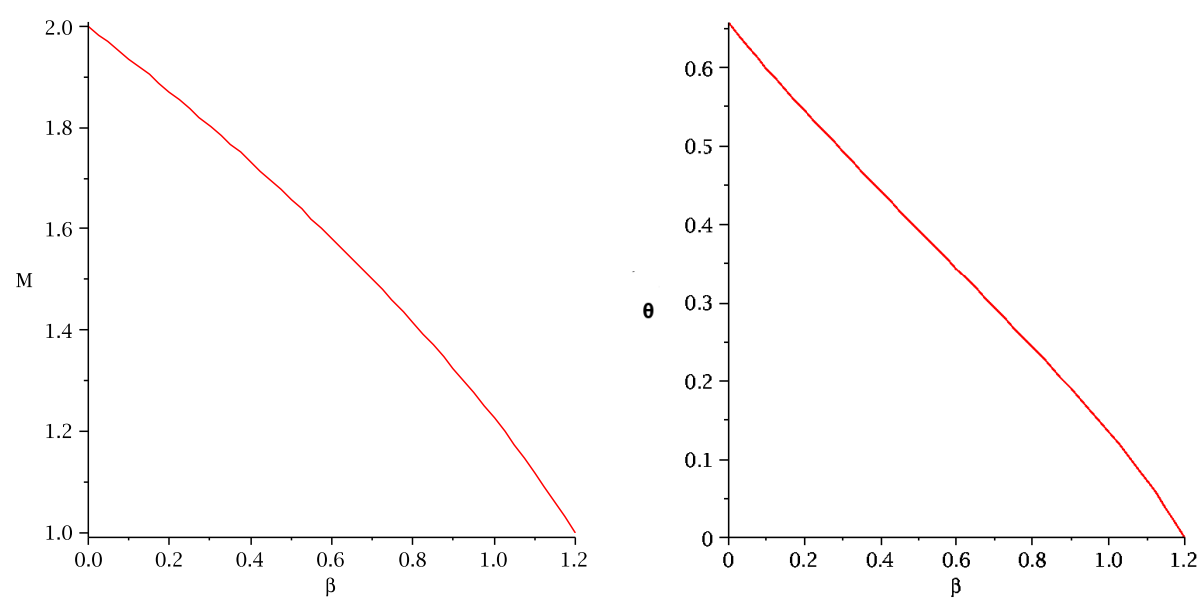

Figure 6: Left: The critical upstream Alfvén Mach number for the existence of intermediate $1 \rightarrow 3$ shocks in function of the upstream $\beta$. Right: The critical upstream $\theta$ number for the existence of intermediate $1 \rightarrow 3$ shocks in function of the upstream $\beta$.

\subsection{Parameter ranges for $1 \rightarrow 3$ shocks.}

The upstream state.

We now search for critical values for the upstream parameters, for which the $\mathrm{RH}$ conditions allow for $1 \rightarrow 3$ shocks. First, note that no $1 \rightarrow 3$ shocks are possible for $\beta>\frac{2}{\gamma}$, since region $(i v)$, as shown in figure (4) then no longer exists.

When, at fixed $\beta$, implicitly taking derivatives of $M$ to $\theta$ on the boundary $\Omega=0$, it can be shown that $\frac{\partial M}{\partial \theta}<0$, for $\theta>0$ and $M>1$, and the other way around: $\frac{\partial M}{\partial \theta}<0$, for $\theta<0$ and $M>1$. Therefore the maximum value of $M$ on $\Omega=0$ is reached when $\theta=0$ (which in figure 4 coincides with point $\mathrm{P}$ ). Hence the maximum value of the upstream Mach number at which intermediate $1 \rightarrow 3$ shocks can be found is reached at $\theta=0$. For varying $\beta$, the left panel of figure (6) shows the maximum value of $M_{1,1-3}$. Therefore $1<M_{1,1-3}<2$.

The maximum value of $\theta_{1}$ for which intermediate $1 \rightarrow 3$ shocks can be found, is reached on the curve traced out by point $Q$ for varying $\beta$ in figure 4 , and thus requires solving the system

$$
\left\{\begin{array}{l}
v_{n}=v_{f} \\
\Omega=0
\end{array}\right.
$$

The analytical expression of the solution is again complicated, but the right panel of figure (6) plots the solution in function of $\beta$. Since this critical value is decreasing for increasing $\beta$, and the limit value for $\beta=0$, equals 0.65633 , we conclude that $-0.65633<\theta_{1,1 \rightarrow 3}<0.65633$.

\section{The downstream state.}

We now search for critical values for the downstream parameters, for which the $\mathrm{RH}$ conditions allow for $1 \rightarrow 3$ shocks.

The downstream region in which $1 \rightarrow 3$ shocks can occur, is region (vii) (as also shown in figure 4 ). Hence, we find the maximum downstream $\theta$ for $1 \rightarrow 3$ shocks to be reached on the curve traced out by point $S$ for varying $\beta$ (as also shown in 


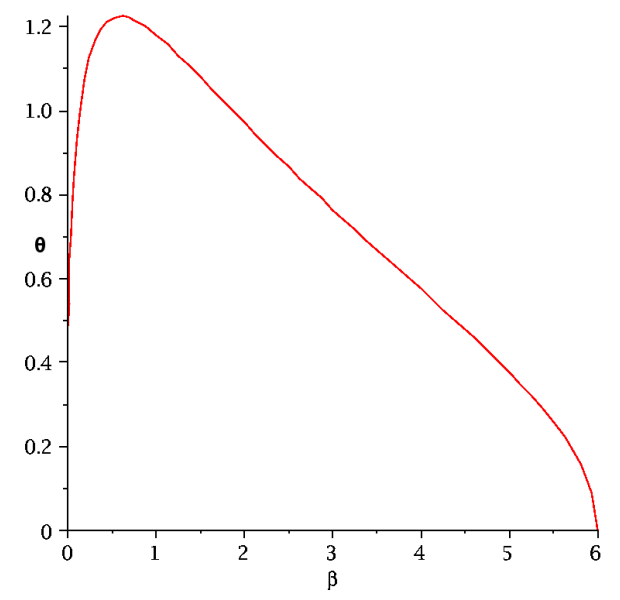

Figure 7: The critical downstream $\theta$ for which $1 \rightarrow 3$ shocks can occur, in function of the downstream $\beta$.

figure 4. Therefore this value can be found by solving

$$
\left\{\begin{array}{l}
M=1 \\
p_{u}=0
\end{array}\right.
$$

We find this critical $\theta$ to be located at

$$
\theta=\sqrt{\frac{(\gamma-1) \beta^{2}+(\gamma-3) \beta-2 \sqrt{\beta(\gamma \beta+\gamma-1)}}{(\gamma-1)(\beta+1)^{2}}},
$$

for $\left.\beta \in] 0, \frac{4}{\gamma-1}\right]$. Note that the maximum value $\frac{1}{\sqrt{\gamma-1}}$ is reached for $\beta=\gamma^{-1}$. Therefore no $1 \rightarrow 3$ shocks are possible for $\theta>(\gamma-1)^{-1 / 2}=0.77460$.

As a bonus, we derived that for $\beta>\frac{4}{\gamma-1}=0$, no $1 \rightarrow 3$ shocks can occur. Figure 7 plots this critical value of $\theta$ in function of $\beta$.

We find the maximum downstream $M$ for $1 \rightarrow 3$ shocks to be reached on the curve related to the intersections labeled as $T$ or $U$ (as also shown in figure 4), depending on the value of $\beta$. At $\beta=0.1$ this maximum downstream value is found to be located at $M=0.94943$, as seen in the top panel of figure (4)..

\subsection{Parameter ranges for $2 \rightarrow 3$ shocks.}

The upstream state.

The maximum value of $M$ at which the $\mathrm{RH}$ conditions allow for $2 \rightarrow 3$ shocks, is reached on the curve related to point $Q$, as shown in figure (4), and can thus be found by solving equations (4.9). The left panel of figure (8) also shows a plot of the Alfvén Mach number on $Q$ for varying $\beta$. Since this value is decreasing for increasing $\beta$, and the limit value for $\beta=0$, equals 1.19615 , we find that $1<$ $M_{1,2 \rightarrow 3}<1.19615$.

The maximum upstream value of $\theta$ at which $2 \rightarrow 3$ shocks can occur is reached on the curve related to point $R$, as shown in figure (4). Hence, we need to solve the following system:

$$
\left\{\begin{array}{c}
p_{u}=0 \\
\Omega=0 .
\end{array}\right.
$$



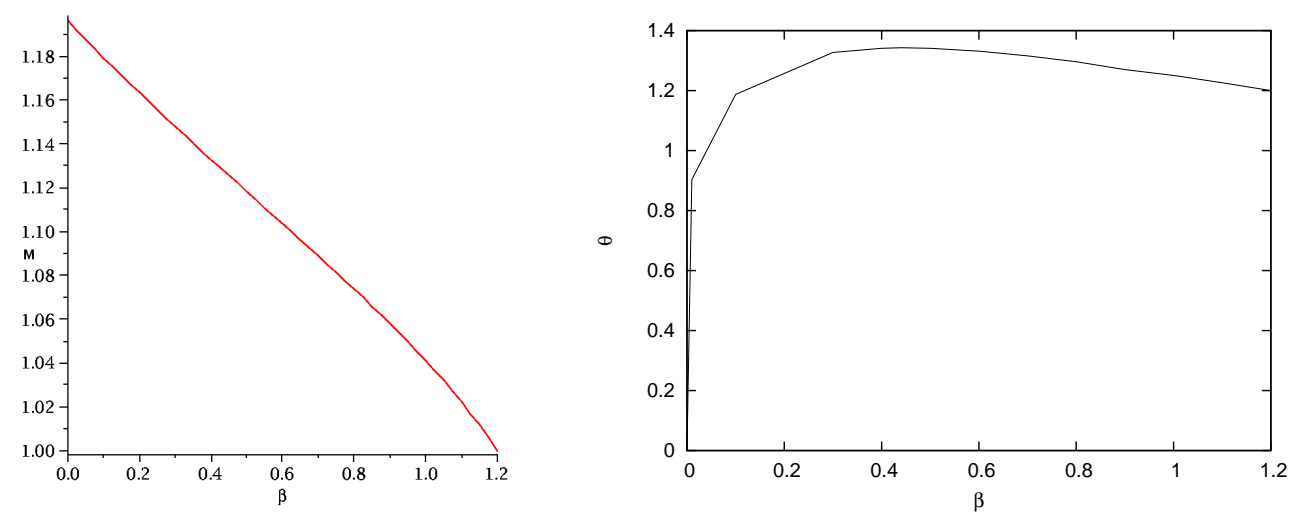

Figure 8: Left: The critical upstream Alfvén Mach number for the existence of intermediate $2 \rightarrow 3$ shocks in function of the upstream $\beta$. Right: The critical value of $\theta$, for the upstream state of an intermediate $2 \rightarrow 3$ shock for varying $\beta$.

A straighforward iteration on $\beta$ shows that the maximum value is reached at $\beta=0.44$, and equals $\theta_{2}=1.34283$, hence $-1.34283<\theta_{2,2 \rightarrow 3}<1.34283$. The variation with $\beta$ is shown in the right panel of figure (8).

The downstream state.

Since the downstream state of a $2 \rightarrow 3$ shock is located in region (vii) or (viii), as also shown in figure (4), and the lower branch of the $p_{u}=0$ surface, starting at $T$ does not cross $M=1$, it follows that there are no limiting values for the downstream $\theta$ of an intermediate $2 \rightarrow 3$ shock.

We find the maximum downstream $M$ for $2 \rightarrow 3$ shocks to be reached on the curve $\mathrm{T}$ or $\mathrm{V}$ (as also shown in figure (4)), depending on the value of $\beta$. Therefore, at $\beta=0.1$ this critical value is found to be located at $M=0.94943$ again.

\subsection{Parameter ranges for $1 \rightarrow 4$ shocks}

The upstream state.

The exact same reasoning we made for the upstream state of an intermediate $1 \rightarrow 3$ shock can be repeated, thus the limiting values for the upstream state of a $1 \rightarrow 4$ shock are exactly the same.

\section{The downstream state.}

We search for critical values for the downstream parameters, for which the RankineHugoniot conditions allow for $1 \rightarrow 4$ shocks. The downstream state of a $1 \rightarrow 4$ shock, must be located in region $(x v i)$.

We first find the minimal value of the Mach number $M$ at which $1 \rightarrow 4$ can occur. A first important observation is that for all $\beta>1.2$, the $(\theta, M)$-parameter space contains a region $(x i v)$, since it can be shown that for all $\beta$, the $p_{u}=0$ curve crosses the $v_{n}=v_{s}$.

At $\beta=\frac{3}{2}$, the $p_{u}=0$ curve crosses the $\Omega=0$ curve in $\theta=0$. Therefore, when $\beta>1.5$, the minimum value of $M$ in region (xiv) is reached at $\theta=0$. When $1.2<\beta<1.5$, this minimum value can be located at point $T$, as labeled in the bottom panel of figure 4 ). 
When $\beta>1.5$, this value is reached on curve $\mathrm{T}$, hence we need to solve

$$
\left\{\begin{array}{c}
p_{u}=0 \\
\theta=0
\end{array}\right.
$$

in order to find the minimum Mach number for $1 \rightarrow 4$ intermediate shocks. For fixed $\beta$, this minimum value is found to be $M=\sqrt{\frac{4-5 \beta+\sqrt{25 \beta^{2}-24 \beta}}{4}}$. This function reaches its minimum at $\beta=1.5$, and the minimal value is 0.5 . It can be shown that for $\beta<1.5$, this minimum value of $M$ is bigger. Therefore $0.5<M_{2,1 \rightarrow 4}<1$.

For fixed $\beta_{2}$, we can also find the maximum value for $\theta_{2}$. Therefore we solve the system

$$
\left\{\begin{array}{l}
p_{u}=0 ; \\
v_{n}=v_{s} .
\end{array}\right.
$$

For $\beta=2$, we find this critical value to be $\theta=0.75604$, as seen in figure (4).

\subsection{Parameter ranges for $2 \rightarrow 4$ shocks}

The upstream state.

The upstream state for an intermediate $2 \rightarrow 4$ shock should be located in region $(v)$. The limiting upstream values for $2 \rightarrow 3$ shocks are exactly the same as the limiting upstream values for $2 \rightarrow 3$ shocks.

The downstream state.

The downstream state of a $2 \rightarrow 4$ shock, must be located in region $(x v)$ or $(x v i)$. Since on $p_{u}=0$, it can be shown that, for fixed $\beta, \frac{\partial M}{\partial \theta}>0$. Therefore $M_{2,2, \rightarrow 4}$ will reach its minimum value in region $(x v i)$, and it equals the minimum value for $M_{2,1 \rightarrow 4}$

The maximum value of $\theta_{2,2 \rightarrow 4}$ is reached on the curve corresponding to point $V$ in figure (4), and for $\beta=2, \theta_{2,2 \rightarrow 4}=1.65654$, as also shown in figure (4).

\section{Conclusion}

Magnetohydrodynamical shocks are governed by the Rankine-Hugoniot jump conditions. These equations can be solved analytically, and doing so essentially reduces to solving a cubic equation. This solution can have one or three real solutions. When there is only one real solution, it corresponds to a fast or a slow magnetoacoustic shock, but when there are three real solutions, also intermediate shock solutions, which cross the Alfvén speed, can be found. Inspired by the time reversal principle from Goedbloed (2008), we revisited the RH shock relations in the frequently employed shock frame, and made the duality visible in the $(\theta, M, \beta)$ state space. Using the thus obtained graphical classification of the state space, augmented with a positive pressure requirement, we derived limiting values for parameters in the shock rest frame at which intermediate shocks can be found.

\section{References}

Akhiezer, A. I., Lyubarskit, G. Ya., Polovin, R. V. 1959 On the stability of shock waves in magnetohydrodynamics. Soviet Phys. -JETP 8, 507511. 
Anderson, J. E. 1963 Magnetohydrodynamical Shock Waves. MIT Press, Cambridge, Massachusetts.

Barmin, A. A., Kulikovskiy, A. G. \& Pogorelov, N. V. 1996 Shockcapturing approach and nonevolutionary solutions in magnetohydrodynamics. J. Comp. Phys. 126, 77-90.

Brio, M. \& Wu, C. C. 1988 An upwind differencing scheme for the equations of ideal magnetohydrodynamics. J. Comp. Phys. 75, 400-422.

Chao, J. K., Lyu, L. H., Wu, B.H., Lazarus, A. J., Chang, T.S. \& LEPPING, R.P. 1993 Observation of an intermediate shock in interplanetary space. J. Geophys. Res. 98, 17443-17450.

Coppi, P. S., Blandford, R. D. \& Kennel, C. F. 1988 On the existance and stability of intermediate shocks ESA SP-285, 381-384

Chu, C. K. \& Taussig, R. T. 1967 Numerical Experiments of Magnetohydrodynamic Shocks and the Stability of Switch-On Shocks. Phys. Fluids 10, 249-256.

De Sterck, H., Low, B. C. \& Poedts, S. 1998 Complex magnetohydrodynamic bow shock topology in field-aligned low- $\beta$ flow around a perfectly conducting cylinder Phys. of Plasmas 11, 4015-4027.

De Sterck, H. \& Poedts, S. 2000 Intermediate Shocks in ThreeDimensional Magnetohydrodynamic Bow-Shock Flows with Multiple Interacting Shock Fronts Phys. Rev. Lett. 84, 5524-5527.

Falle, S. A. E. G. \& Komissarov, S. S. 1997 On the existence of intermediate shocks. Mon. Not. R. Astron. Soc. 123, 265-277.

Falle, S. A. E. G. \& Komissarov, S. S. 2001 On the inadmissibility of non-evolutionary shocks. J. Plasma Phys. 65, 29-58.

FEng, H. \& WANG, J. M. 2008 Observations of a $2 \rightarrow 3$ Type Interplanetary Intermediate Shock. Solar Phys. 247, 195-201.

Germain, P. 1960 Shock Waves and Shock-wave structure in magneto-fluid dynamics. Rev.Mod.Phys. 32, 951-958.

GLimM, J. 1965 Solutions in the large for nonlinear hyperbolic systems of equations. Comm. Pure Appl. Math 41, 569-590

Goedbloed, H. \& Poedts, S. 2004 Principles of Magnetohydrodynamics With Applications to Laboratory and Astrophysical Plasmas. Cambridge University Press.

Goedbloed, J.P. 2008 Time reversal duality of magnetohydrodynamical shocks. Phys. Plasmas 15, 062101

Gombosi, T. I. 1998 Physics of the Space Environment. Cambridge University Press.

De Hoffmann, F. And Teller, E. 1950 Magneto-Hydrodynamic Shocks. Phys. Rev. 80, 692-703.

Jeffrey, A. \& TANiuti, T. 1964 Nonlinear Wave Propagation. Academic Press, New York.

Kennel, C. F., Blanford, R. D. \& Coppi, P. 1989 MHD intermediate shock discontinuities. I - Rankine-Hugoniot conditions. J. Plasma Phys. 42, 219-319.

LAx, P. D. 1957 Hyperbolic System of Conservation Laws II. Comm. Pure Appl. Math. 10, 537-566.

Liberman, M. A. \& Velikhovich, A. L. 1986 Physics of Shock Waves in Gases and Plasmas. Springer 
Myong, R. S. \& Roe, P. L. 1997 Shock waves and rarefaction waves in magnetohydrodynamics. Part 1. A model system. J. Plasma. Phys. 58, 485. Myong, R. S. \& Roe, P. L. 1997 Shock waves and rarefaction waves in magnetohydrodynamics. Part 2. The MHD system. J. Plasma. Phys. 58, 521. Sturtevant, B. 1987 Shock Tubes and Waves. VCH Verlag, Berlin.

TODD, L. 1965 Evolution of switch-on and switch-off shocks in a gas of finite electrical conductivity. J. Fluid Mech. 24, 597-608.

Whang, Y. C., Zhou J., Lepping, R. P., Szabo, A., Fairfield, D., Kukobun, S., Ogilvie, K. W., Fitzenreiter R. 1998 Double discontinuity : A compound structure of slow shock and rotational discontinuity $J$. Geophys. Res. 103 6513-6520.

Wu, C. C. 1987 On MHD intermediate shocks. Geophys. Res. Lett. 14, 668671 .

Wu, C. C. 1988 The MHD intermediate shock interaction with an intermediate wave: Are intermediate shocks physical?. J. Geophys. Res. 93(A2), 987-990.

Wu, C. C. 1990 Formation, structure, and stability of MHD intermediate shocks. J. Geophys. Res. 95(A6), 8149-8175 